\title{
SEM Images Obtained with an Energy and Takeoff Angle Selective Detector*
}

\author{
Takeshi Otsuka, ${ }^{\dagger}$ Motohiro Nakamura, Ken-ichi Yamashita, Kazuhiro Honda, and Shin-ichi Kitamura \\ JEOL Ltd., 1-2 Musashino 3-chome, Akishima, Tokyo 196-8558, Japan
}

Felix Timischl and Masato Kudo

JEOL Technics Ltd., 6-38 Musashino 2-chome, Akishima, Tokyo 196-0021, Japan

(Received 15 January 2014; Accepted 7 April 2014; Published 21 June 2014)

\begin{abstract}
SEMs are usually equipped with a backscattered electron detector and a secondary electron detector. In addition to these, alternative detectors have recently been employed to obtain images with additional information content. These detectors are designed to detect electrons emitted within a certain predefined range of energy and takeoff angle. However, no attempt has ever been made to design a detector that allows direct detection of electrons in a user-defined energy and takeoff angle range. In this study, an electron detector was designed and experimentally manufactured to detect electrons emitted in a defined, variable range of energy and takeoff angle. With this " $E-\theta$ detector" a set of images was taken to obtain electrons in two distinct ranges of energy and two distinct ranges of takeoff angle. These images were compared with those obtained by ordinary secondary and backscattered electron detectors. As an application example, clear contrast originating from crystal orientation of a spherical tungsten single crystal was observed in an image composed of high takeoff angle secondary electrons.
\end{abstract}

[DOI: $10.1380 /$ ejssnt.2014.279]

Keywords: Scanning electron microscopy (SEM); Surface electronic phenomena (work function, surface potential, surface states, etc.); Secondary electron emission; Single crystal surfaces; Curved surfaces; Tungsten; Titanium

\section{INTRODUCTION}

Scanning electron microscopes (SEMs) are usually equipped with two types of detectors: secondary and backscattered electron detectors. The former gives secondary electron images (SEI) rich in topographic information $[1,2]$, whereas the latter gives backscattered electron images (BEI) rich in material information [3]. Recently, alternative detectors have been employed for additional information acquisition. As an example, the JSM$7800 \mathrm{~F}$ field emission SEM allows installation of the following four different detector types: the upper electron detector (UED), the upper secondary electron detector (USD), the backscattered electron detector (BED), and the lower electron detector (LED). All give different contrast and additional information about the observed specimen. They are in fact designed to collect useful information about the sample surface condition. To further improve the detector design, it is important to understand the relationship between image contrast and the contrast forming properties (i.e. energy and takeoff angle) of the detected electrons [4].

We are planning to conduct a series of studies with the objective of acquisition and evaluation of images obtained by electrons with defined ranges of energy and takeoff angle. In this study, as a first step, we designed and manufactured a detector for electrons emitted from a specimen within two distinct ranges of energy and takeoff angle, respectively. Using this so called " $E-\theta$ detector", we acquired images with four different combinations of energy and takeoff angle ranges. These images were compared with conventional SEIs and BEIs.

*This paper was presented at 9th International Symposium on Atomic Level Characterizations for New Materials and Devices, Sheraton Kona, Hawaii, U. S. A., December 2-6, 2013.

†Corresponding author: tootsuka@jeol.co.jp

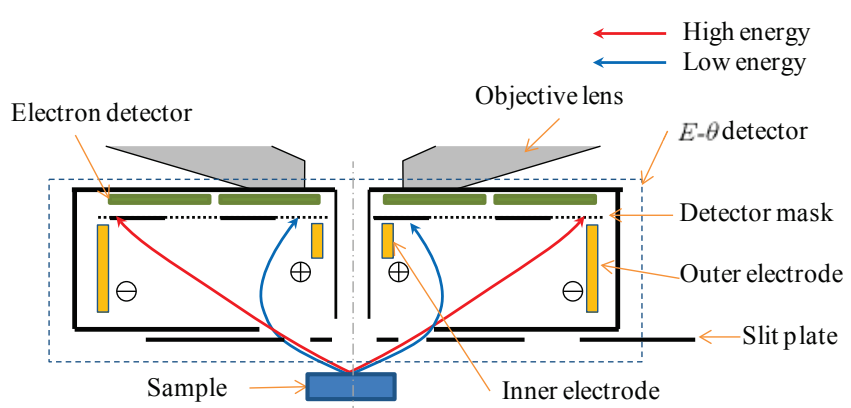

(a) Low angle range detection setup

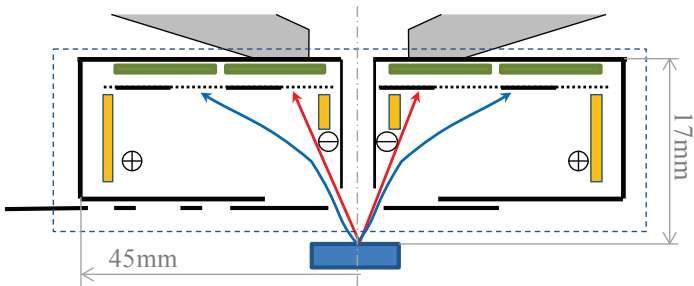

(b) High angle range detection setup

FIG. 1: A schematic diagram of the newly designed $E-\theta$ detector. Two types of slits are placed at the bottom of the detector cage, serving as takeoff angle selectors. (a) A broken concentric ring slit with a small center hole for the low angle range, and (b) a single hole slit for the high angle range.

\section{II. $E-\theta$ DETECTOR}

Figure 1 shows a schematic diagram of the newly designed electron detector: the $E-\theta$ detector, which can detect electrons emitted from a specimen within a specified range of energy and takeoff angle. The $E-\theta$ detector consists of a slit plate, inner and outer electrodes in a cage, and an electron detector.

The slit plate is placed below the bottom face of the 


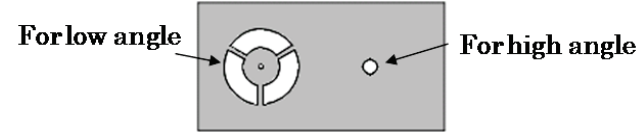

FIG. 2: The shape of the two types of slits in the slit plate as shown in Fig. 1.

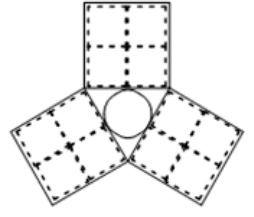

(a)

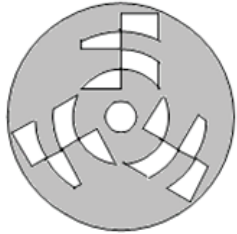

(b)
FIG. 3: (a) The arrangement of the Si-photodiode detectors and (b) the mask for the detectors.

$E-\theta$ detector cage. It serves as a selector of takeoff angle and has two types of slits: a single hole slit for a high angle range and a broken concentric ring slit with a small center hole for a low angle range as shown in Fig. 2. Takeoff angle range selection is done mechanically by sliding the slit plate as shown in Fig. 1. In the present study, the single hole slit was designed to cover the range between 60 and 70 degrees (Fig. 1(b)), whereas the broken concentric ring slit covers the range between 20 and 30 degrees (Fig. 1(a)). The takeoff angle is measured from the horizontal direction parallel to the sample surface.

Electron energy range selection is done by applying voltage to the inner and outer electrodes in the cage. In the case of the low angle range, positive and negative voltages are applied to the inner and outer electrode, respectively, as shown in Fig. 1(a), so that electrons are deflected towards the inner electrode, where lower energy results in a higher deflection angle. In the case of the high angle range, the polarity of the applied voltage is reversed as shown in Fig. 1(b), so that electrons are deflected towards the outer electrode. If a series of electron detectors are arranged like concentric rings with different diameters and placed below the upper confinement of the cage, each ring detector may collect electrons with a specific range of energy determined by the voltages applied to the two electrodes. In the present study, a Si-photodiode (SiPD) was used as the electron detecting element. Instead of a series of concentric ring detectors, three square shaped SiPD detectors, each consisting of four segments, were arranged as shown in Fig. 3(a). In addition, a detector mask was prepared and placed in front of the electron detectors to separately detect two different energy ranges. The shape of the mask is shown in Fig. 3(b). In the case of the low angle range, the six inner openings collect one energy range, and the remaining six outer openings collect another energy range, whereas in the case of the high angle range, three inner openings collects one energy range and the remaining nine openings collect another energy range. It has to be noted that the two energy ranges can be de-

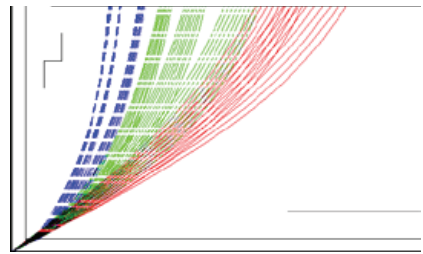

\section{- High energy \\ Intermed iate energy \\ -- - Low energy}

(a)

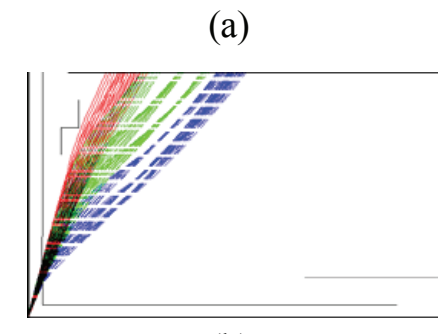

(b)
FIG. 4: Computed Electron trajectories using the Charge Simulation Method. Three energy ranges were designed for discrimination: a low energy range, an intermediate range, and a high range, referring to the energy of the electrons emitted from the specimen surface. Analogously, two takeoff angle ranges were assumed: (a) a low takeoff angle range, and (b) a high takeoff angle range.

tected simultaneously. To enhance electron detection, a metal mesh was placed in front of the SiPD detectors, and a voltage of $2 \mathrm{keV}$ was applied between the detectors and the mesh.

Electron trajectories resulting from an incident electron beam with $3 \mathrm{kV}$ were calculated by the use of the Charge Simulation Method (CSM). The results are shown in Figs. 4(a) and 4(b). In these figures, the bold vertical line at the left represents the optical axis of the incident electron beam, whereas the bold horizontal line at the bottom represents the sample surface. The thin vertical line at the left and the thin horizontal line at the bottom represent the $E-\theta$ detector cage. The thin horizontal line at the top represents the metal mesh and two other thin bent lines represent the inner and outer electrodes. Fig. 4(a) shows the results in the low takeoff angle range between 20 and 30 degrees, and Fig. 4(b) shows those in the high takeoff angle range between 60 and 70 degrees. In the former case, $-200 \mathrm{~V}$ and $800 \mathrm{~V}$ were applied to the inner and outer electrode respectively, whereas in the latter case, $500 \mathrm{~V}$ and $-1300 \mathrm{~V}$ were applied to the inner and outer electrode, respectively. In both cases, the trajectories were calculated for three ranges of energy a low energy range: $10 \mathrm{eV}, 20 \mathrm{eV}$ and $50 \mathrm{eV}$; an intermediate energy range: $100 \mathrm{eV}, 200 \mathrm{eV}$ and $500 \mathrm{eV}$; and a high energy range: $1 \mathrm{keV}$ and $2 \mathrm{keV}$. In the former case, the emitted electrons were deflected towards the inside whereas in the latter case, they were deflected outside, as shown in Fig. 4. In both cases, the amount of their deflection became larger with decrease in energy. Separated electron detectors were placed at the position of the metal mesh in order to select emitted electrons with a defined range of energy. In fact, the experimental setup was determined by the calculated trajectories.

A fraction of the backscattering electrons emitted from the specimen does not reach the detector directly, but 

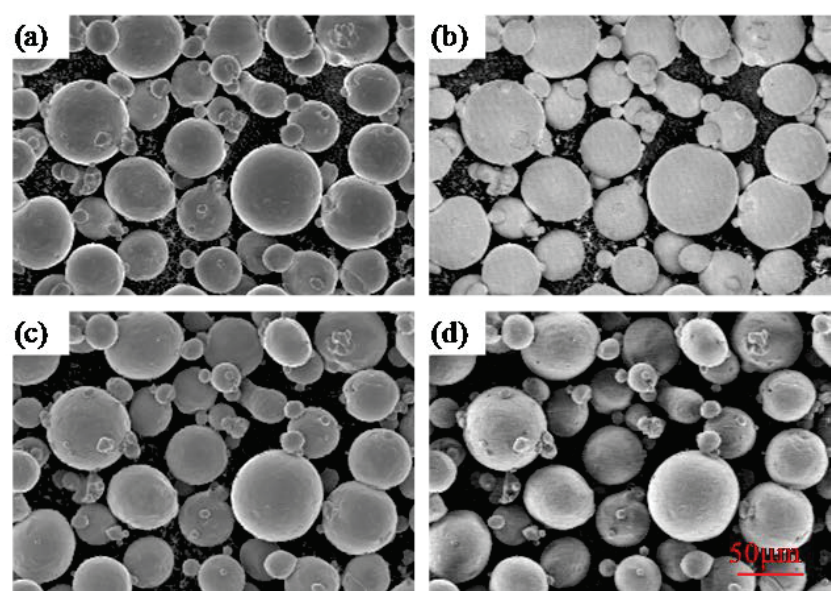

FIG. 5: A set of four images of spherical titanium particles obtained with the $E-\theta$ detector. (a) I: low energy and high angle range, (b) II: high energy and high angle range, (c) III: low energy and low angle range and (d) IV: high energy and low angle range.
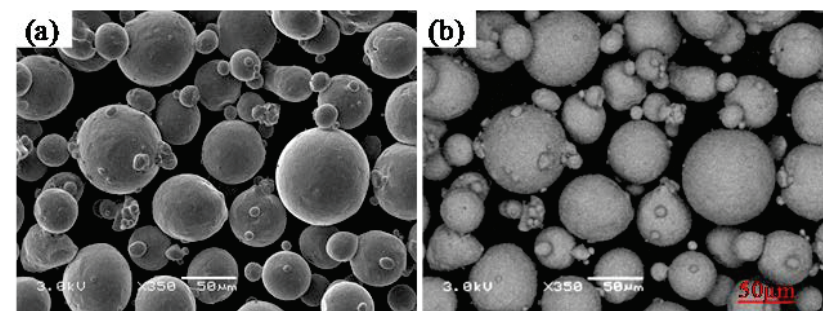

FIG. 6: Conventional images of the same field of view as in Fig. 5 taken with a JSM-6460 SEM. (a) Secondary electron image and (b) back-scattered electron image.

strikes the detector mask or the electrodes, and produces additional secondary electrons. Electron trajectory simulation shows that the majority of these scattering secondary electrons do not reach the detector. The small fraction of scattering secondary electrons that can be detected carries the energy information of the original backscattered electrons, since the scattering process occurs after energy separation, and therefore does not influence the energy separation result.

\section{RESULTS AND DISCUSSION}

Using the $E-\theta$ detector, we obtained images of two different specimens (titanium particle powder and a spherical tungsten single crystal) with an incident electron beam of $3 \mathrm{keV}$ at four different combinations of emission energy and angle (I: $<0.2 \mathrm{keV}, 60-70$ degrees; II: $0.2-3 \mathrm{keV}, 60-$ 70 degrees; III: $<0.2 \mathrm{keV}, 20-30$ degrees; IV: $0.2-3 \mathrm{keV}$, 20-30 degrees). In addition to the four images by the $E-\theta$ detector, corresponding conventional images - SEI and BSI - were taken with the same incident electron beam of $3 \mathrm{keV}$ with a JSM-6460 SEM. Roughly speaking, the conventional SEI corresponds to the $E-\theta$ detector image taken at the condition III, whereas the BSI corresponds
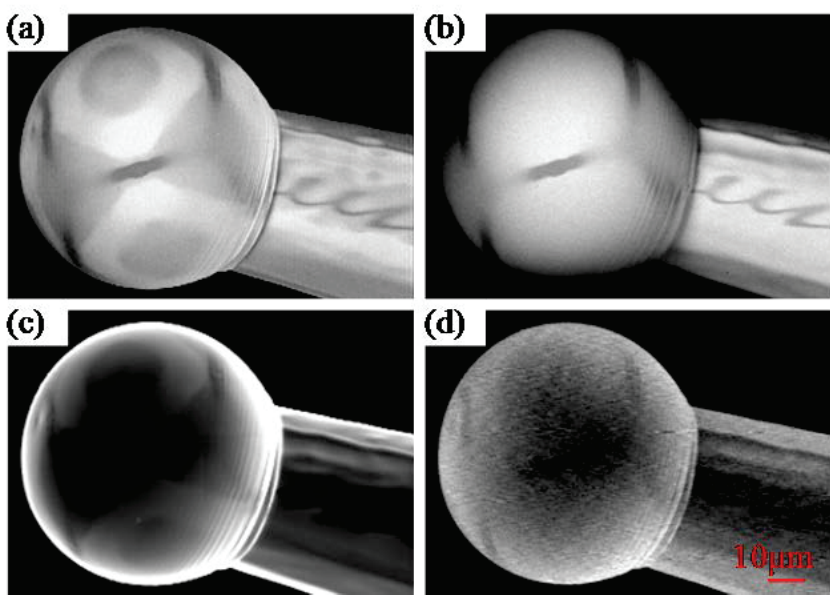

FIG. 7: A set of four images of a spherical tungsten single crystal obtained with the $E-\theta$ detector. (a) I: low energy and high angle range, (b) II: high energy and high angle range, (c) III: low energy and low angle range and (d) IV: high energy and low angle range.
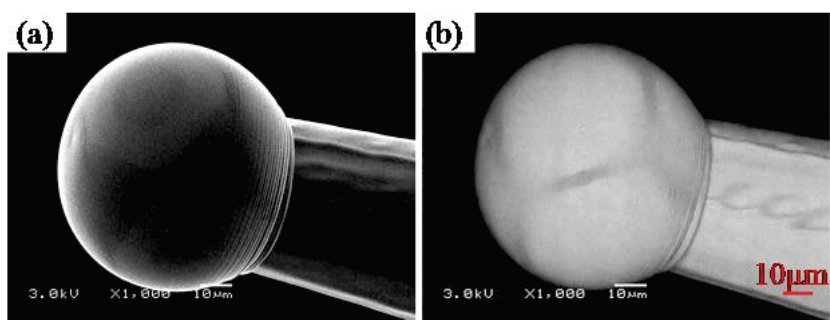

FIG. 8: Conventional images of the same field of view as in Fig. 7 taken with a JSM-6460 SEM. (a) Secondary electron image and (b) back-scattered electron image.

to the $E-\theta$ detector image at the condition II.

\section{A. Images of spherical titanium particles}

A powder specimen of spherical titanium particles with diameters of the order of $50 \mu \mathrm{m}$ was used in this sec-

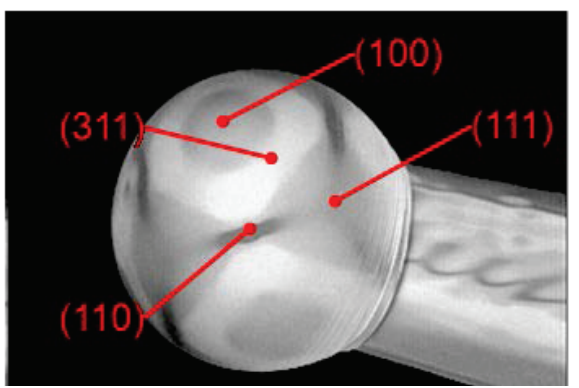

FIG. 9: Several crystal orientations of a spherical tip of a body centered cubic tungsten single crystal deduced from the symmetry of the observed contrast patterns. 
TABLE I: Work function dependence on crystal face orientation in the case of tungsten [5].

\begin{tabular}{cc}
\hline \hline Crystal orientation & Work function $(\mathrm{eV})$ \\
\hline$(110)$ & 5.25 \\
$(100)$ & 4.63 \\
$(111)$ & 4.47 \\
$(611)$ & 4.3 \\
$(311)$ & 4.18 \\
\hline \hline
\end{tabular}

tion. The obtained set of images is shown in Fig. 5. The corresponding conventional images, the SEI and BEI, are shown in Fig. 6. Figures 5(b) and 5(d) are images taken at the condition of II and IV with the same high energy range but different takeoff angle ranges (60-70 degrees and 20-30 degrees). An illumination effect in the lower takeoff angle case is clearly visible in Fig. 5(d); for instance, small particles at deeper positions, especially near to large ones, show dark contrast. Figures 5(a) and 5(c) are images taken at the condition of I and III with the same low energy range but different takeoff angle ranges (60-70 degrees and 20-30 degrees). In these figures, the periphery of some of the particles shows bright contrast. This periphery contrast in Fig. 5(c) is caused of the edge effect. The particles influenced by the illumination effect in Fig. 5(d) do not show the periphery contrast in Fig. 5(c).

\section{B. Images of a spherical tungsten single crystal}

Another set of images of a spherical tungsten single crystal (the tip of a filament with a diameter of about $50 \mu \mathrm{m}$ ) is shown in Fig. 7. The corresponding conventional images, the SEI and BEI are also shown in Fig. 8.

A distinct contrast, different from that explained in the previous section A, is observed in Figs. 7 and 8, especially in Fig. 7(a). This type of contrast consists of a pattern corresponding to the symmetry in accord with body-centered cubic tungsten - the 4-fold, 3-fold and 2 -fold symmetry. From the symmetry of each pattern, orientations of the crystal face are identified as shown in Fig. 9. It has to be noted that this contrast arises from the difference of the work function, which depends on the orientation of the crystal face. The work functions on the crystal face orientations are listed in Table I [5]. The table shows that the 110-direction corresponds to the largest work function value (which gives the darkest contrast), whereas 311-direction corresponds to the smallest work function value (which gives the brightest contrast). The correlation strongly suggests that a larger value of the work function may reduce the intensity of the emitted secondary electrons which results in a darker contrast area.

This is the first study to observe clear contrast originating from work function differences determined by crystal face orientation. The success of this observation is partly due to the newly manufactured $E-\theta$ detector which can produce images using secondary electrons in a high takeoff angle range, and partly due to the observed tungsten specimen with relatively clean dependence of the work function in each orientation.

\section{CONCLUSIONS}

A series of systematic studies on image formation has been done firstly due to emitted electrons with defined energy and takeoff angle.

In this study, the $E-\theta$ detector was designed and manufactured with the objective of acquisition of a set of four images with two different ranges of energy and takeoff angle. The detector setup was applied to two different specimens: spherical titanium particles and a spherical tungsten single crystal. These images were compared to those obtained by conventional SEI and BEI detectors. All images obtained with the $E-\theta$ detector gave different contrasts, which prove the usefulness of the $E-\theta$ detector and the necessity of the present study.

A novel result in this study is the clean visualization of the crystal face orientations of the spherical tungsten single crystal specimen. This observation could be realized with the $E-\theta$ detector by selection of secondary electrons with high takeoff angle.
[1] M. Kotera, T. Kishida, and H. Suga, Scanning Microscopy Supplement 4, 111 (1990).

[2] Y. Lin and D. C. Joy, Surf. Interface Anal. 37, 895 (2005).

[3] M. D. Ball and D. G. McCartney, J. Microscopy 124, 57 (1981).
[4] J. Cazaux, N. Kuwano, and K. Sato, Ultramicroscopy 135, 43 (2013).

[5] H. B. Michelson, J. Appl. Phys. 48, 4729 (1977). 